\title{
Are the rib fracture score and different computed tomography measures of obesity predictors for mortality in patients with rib fractures? A retrospective cohort study
}

\author{
Thorsten Jentzsch ${ }^{1,2} \mathbb{D} \cdot$ Valentin Neuhaus $^{1} \cdot$ Burkhardt Seifert $^{3} \cdot$ Rudolf M. Moos $^{4} \cdot$ Hans-Peter Simmen $^{1}$. \\ Christoph E. W. Schmitz ${ }^{1}$. Clément M. L. Werner ${ }^{1}$
}

Received: 8 May 2020 / Accepted: 24 August 2020 / Published online: 6 September 2020

(C) The Author(s) 2020

\begin{abstract}
Background There is missing knowledge about the association of obesity and mortality in patients with rib fractures. Since the global measure of obesity (body mass index [BMI]) is often unknown in trauma patients, it would be convenient to use local computed tomography (CT)-based measures (e.g., umbilical outer abdominal fat) as a surrogate. The purpose of this study was to assess (1) whether local measures of obesity and rib fractures are associated with mortality and abdominal injuries and to evaluate (2) the correlation between local and global measures of obesity.

Materials and methods A retrospective cohort study included all inpatients with rib fractures in 2013. The main exposure variable was the rib fracture score (RFS) (number of rib fractures, uni- or bilateral, age). Other exposure variables were CTbased measures of obesity and BMI. The primary outcome (endpoint) was in-hospital mortality. The secondary outcome consisted of abdominal injuries. Sex and comorbidities were adjusted for with logistic regression.

Results Two hundred and fifty-nine patients (median age 55.0 [IQR 44.0-72.0] years) were analyzed. Mortality was 8.5\%. RFS $>4$ was associated with $490 \%$ increased mortality $\left(\mathrm{OR}_{\text {adjusted }}=5.9,95 \%\right.$ CI $\left.1.9-16.6, p=0.002\right)$. CT-based measures and BMI were not associated with mortality, rib fractures or injury of the liver. CT-based measures of obesity showed moderate correlations with BMI (e.g., umbilical outer abdominal fat: $r=0.59, p<0.001$ ).

Conclusions RFS $>4$ was an independent risk factors for increased mortality. Local and global measures of obesity were not associated with mortality, rib fractures or liver injuries. If the BMI is not available in trauma patients, CT-based measures of obesity may be considered as a surrogate.
\end{abstract}

Keywords Adiposity $\cdot$ Bone $\cdot$ Computed tomography $\cdot$ Diagnosis $\cdot$ Fat mass

Christoph E. W. Schmitz and Clément M. L. Werner have contributed equally.

Thorsten Jentzsch

thorsten.jentzsch@balgrist.ch

Valentin Neuhaus

valentin.neuhaus@usz.ch

Burkhardt Seifert

seifert@ifspm.uzh.ch

Rudolf M. Moos

rudolf.moos@usz.ch

Hans-Peter Simmen

hanspeter.simmen@usz.ch

Christoph E. W. Schmitz cewschmitz@gmail.com
Clément M. L. Werner

clement.werner@hirslanden.ch

1 Department of Traumatology, University Hospital Zurich, University of Zurich, Ramistrasse 100, 8091 Zurich, Switzerland

2 Department of Orthopaedics, Balgrist University Hospital, University of Zurich, Zurich, Switzerland

3 Department of Biostatistics, Epidemiology, Biostatistics and Prevention Institute, University of Zurich, Zurich, Switzerland

4 Medical Directorate, University Hospital Zurich, University of Zurich, Zurich, Switzerland 


\section{Introduction}

\section{Background}

Rib fractures are commonly (4-12\%) found in trauma patients and are associated with a relevant death rate (12\%) [1]. Obesity is commonly measured with the body mass index (BMI) and can also be examined by measuring the waist circumference, both of which can predict all-cause mortality $[2,3]$.

There are studies that have shown that obesity measured with the BMI is a risk factor for peri-traumatic mortality and complications [4], but others have reported that obesity may be protective of certain injuries (e.g., hip fractures) due to a cushioning effect [5]. However, there is a lack of literature about the association of obesity and mortality in patients with rib fractures. One study reported that the BMI is associated with increased incidence rates for multiple rib fractures [6]. An increased incidence of truncal injuries has also been associated with rib fractures [7]. Although multiple rib fractures have been associated with increased mortality in trauma patients and osteoporotic males, there is evidence that rib fractures are not associated with mortality in postmenopausal women and non-stratified patient cohorts [8-13].

The BMI is often not obtainable in trauma patients and the waist circumference is not commonly evaluated. This is due to the facts that severely injured patients often arrive in a supine position, most weight scales would require an upright position, and trauma patients mostly remain immobilized during the initial evaluation. Recently, other computed tomography (CT)-based anthropometric measures of the thorax and abdomen have been introduced as alternative ways to assess obesity [14-18]. Since patients with chest trauma and rib fractures are often evaluated with CT scans, it seems inviting to consider these new tools as alternative ways of obesity assessment and in the prediction of injury risk as well as mortality.

The objectives of this study were to assess whether rib fractures and different measures of obesity are associated with certain truncal injuries and higher mortality. Rib fractures were assessed with the rib fracture score (RFS), which was first described by Easter and is based on the number and side of rib fractures as well as age, [8, 19, 20]. The correlation between the BMI and CT-based measurements of obesity was also assessed. CT-based measurements of adiposity consist of two subcutaneous and two intra-abdominal distances at the level of the subxiphoid and umbilicus.

\section{Methods}

\section{Study design}

From a cohort of 2829 patients that were treated at the authors' institution, this retrospective cohort study included all inpatients $\geq 18$ years with rib fractures that were treated (discharged) at a level 1 trauma center between January 2013 and December 2013. There were no exclusion criteria. The study was approved by the local ethics committee (Kantonale Ethikkommission Zürich, KEK-ZH-Nr.: 2014-0285) without the need for informed consent due to the retrospective nature of this study using a large dataset.

\section{Exposure and outcome variables}

The main exposure variable was the RFS. Other exposure variables were CT-based measures of obesity and placement of a chest tube. The primary outcome variable (endpoint) was in-hospital mortality. Secondary outcomes (endpoints) were abdominal injury with their respective subcategories (liver, spleen, duodenum, colon, kidney, and bladder). A priori confounders consisted of sex and comorbidity.

\section{Data acquisition and measurements}

An independent investigator (senior resident), who was blinded to the data mentioned above, acquired four axial plane CT-based measures of obesity. CT scans were acquired on a dual-source CT scanner (Somatom Definition, Siemens Healthcare, Forchheim, Germany) and images were evaluated using an IMPAX client (version 6.5.5.1544; AGFA HealthCare Corp., Greenville, SC, USA) [16]. The subxiphoid outer abdominal fat (SOAF) was defined as the subcutaneous anterior abdominal wall diameter from the rectus sheath to the cutis just below the xiphoid process $[14,15$, 17] (Fig. 1a). The subxiphoid peritoneal fat (SPF) was determined as the intraabdominal distance between the liver and the rectus sheath just below the xiphoid process $[14,15]$ (Fig. 1b). The umbilical outer abdominal fat (UOAF) was defined as the subcutaneous anterior abdominal wall diameter from the rectus sheath to the cutis at the level of the umbilicus [16] (Fig. 1c). The umbilical visceral fat (UVF) was determined as the distance between the anterior wall of the aorta and the rectus sheath at the level of the umbilicus (Fig. 1d).

The database of the hospital was searched for all patients with at least one rib fracture, coded as S22.31, 


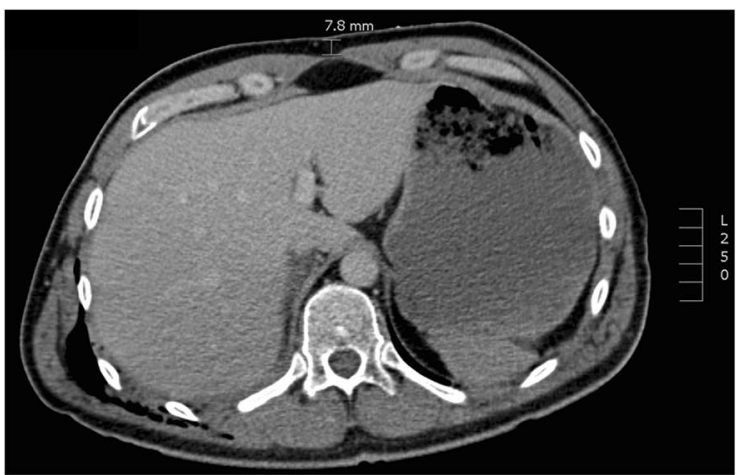

A

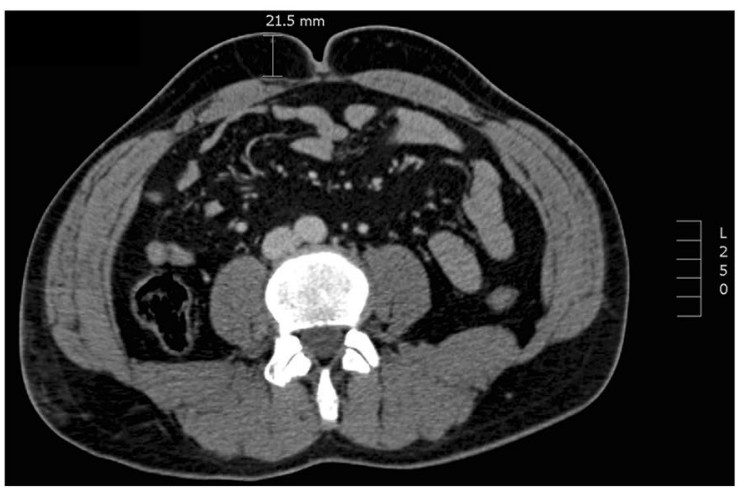

C

Fig. 1 Computed tomography (CT)-based measurements. a Subxiphoid outer abdominal fat (SOAF) on an axial plane of a CT scan. b Subxiphoid peritoneal fat (SPF) on an axial plane of a CT scan.

\section{S22.32, S22.40, S22.41, S22.42, S22.43, S22.44, or S22.5} according to the World Health Organization's International Statistical Classification of Diseases and Related Health Problems (10th Revision, German Modification, Version 2010) [21, 22]. This search provided several epidemiological patient data, which included sex, age, BMI, the presence of comorbidities (including $>40$ diseases, such as coronary heart disease, diabetes mellitus, osteoporosis, neoplastic disease, and depression), death, thoracic injuries, abdominal injuries, and injury severity score (ISS). Thoracic injuries were grouped into isolated rib fracture, and multiple rib fractures of 2,3 or $\geq 4$ ribs. Abdominal injuries consisted of overall intraabdominal injuries, which were further split into injuries of the liver, spleen, duodenum, colon, kidney, and bladder.

The RFS was calculated based on the previously published formula 'number of rib fractures $\times$ side + age category', where the side was categorized into a binary variable (unilateral $=1$ and bilateral $=2$ ) and age was categorized into five categories $(<50=0,50-60=1$, $61-70=2,71-80=3$, and $>80=4)[8]$.

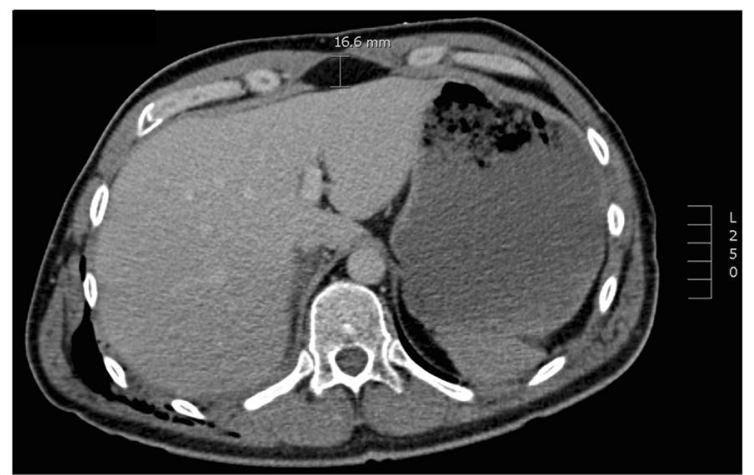

B

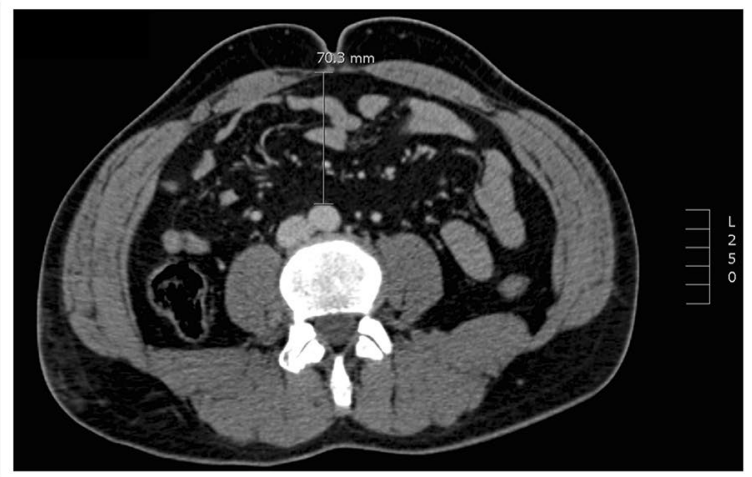

D

c Umbilical outer abdominal fat (UOAF) on an axial plane of a CT scan. d Umbilical visceral fat (UVF) on an axial plane of a CT scan

\section{Statistics}

Data were tested for distribution with the skewness and kurtosis test for normality. They were non-normally distributed and, therefore, the median and interquartile range (IQR) are provided. To account for some missing data within each category, the absolute number of patients and the corresponding percentage are given in the tables. The Chi-squared test was used for the unadjusted analysis of categorical data. The Wilcoxon rank sum test was employed for continuous data. Spearman rank correlation was used to assess correlations between continuous data. For the main outcome, a logistic regression model was implemented to adjust for the a priori confounders sex and comorbidities and used the Wald test for comparison. For the evaluation of the level of obesity, only sex was included as a confounder, since the number of deaths and the incidence of diseases of interest were low. The BMI and CT-based measurements of obesity were categorized into binary variables based on their respective median. Unadjusted and adjusted odds ratios (OR) and the 
corresponding $95 \%$ confidence intervals (CI) are given. A receiver operating characteristic (ROC) curve was performed to assess potential cut-off points. BMI was also stratified according to the most commonly reported cutoff value in the literature $\left(\geq 30 \mathrm{~kg} / \mathrm{m}^{2}\right)$ [5]. Due to multiple testing, the significance level was set at $1 \%$. Analyses were performed with Stata (version 13.1/IC; StataCorp, College Station, TX, USA).

\section{Results}

\section{Participants}

Two hundred and fifty-nine patients were analyzed. There were $172(66.4 \%)$ males and the median age was 55.0 (IQR 44.0-72.0) years. The median RFS was 4 (IQR 3-7). One hundred and forty-six (56.4\%) patients had comorbidities. The median BMI was 25.1 (IQR 23.1-27.8) $\mathrm{kg} / \mathrm{m}^{2}$. The median values of the SOAF, SPF, UOAF, and UVF were 9.4 (IQR 23.1-27.8) mm, 12.5 (IQR 8.9-16.2) mm, 19.5 (13.5-25.0) mm, and 59.0 (IQR 44.0-25.0) mm. Thirty-eight $(14.7 \%)$ patients had an intraabdominal injury, of which 11 (4.3\%) were a liver injury. Twenty-two (8.5\%) patients died.

\section{Obesity and mortality}

The BMI was higher in males (25.7 [IQR 23.9-29.0] versus [vs] 24.2 [21.1-26.8] kg/m², $p=0.002$ ) (Table 1). It was not increased in patients with comorbidity. No association between BMI and mortality (24.4 [IQR 23.5-25.2] vs 26.1 [IQR 22.9-28.0], $p=0.59$; AUC 0.39 [0.31-0.47]) or abdominal injury were observed. Even when choosing a cut-off point of $\geq 30 \mathrm{~kg} / \mathrm{m}^{2}$, there were no differences in
Table 1 Association of outcome variables and body mass index (BMI)

\begin{tabular}{|c|c|c|c|c|c|c|}
\hline \multirow[t]{2}{*}{ Variable } & \multirow[t]{2}{*}{ Category } & \multicolumn{4}{|c|}{ Body mass index (BMI) $(n=146)$} & \multirow[t]{2}{*}{$p$ value* } \\
\hline & & Median & IQR & $n$ & $\%$ & \\
\hline \multirow[t]{2}{*}{ Sex } & Female & 24.2 & $21.1-26.8$ & 46 & 31.5 & \multirow[t]{2}{*}{0.002} \\
\hline & Male & 25.7 & $23.9-29.0$ & 100 & 68.5 & \\
\hline \multirow[t]{2}{*}{ Age (years) } & $\leq 55$ & 24.9 & $23.1-27.8$ & 81 & 55.5 & \multirow[t]{2}{*}{0.49} \\
\hline & $>55$ & 25.6 & $23.4-28.1$ & 65 & 44.5 & \\
\hline \multirow[t]{2}{*}{ Comorbidity } & No & 24.6 & $22.5-27.2$ & 65 & 44.5 & \multirow[t]{2}{*}{0.04} \\
\hline & Yes & 26.1 & $23.9-28.4$ & 81 & 55.5 & \\
\hline \multirow[t]{2}{*}{ Death } & No & 25.2 & $22.9-28.0$ & 144 & 98.6 & \multirow[t]{2}{*}{0.59} \\
\hline & Yes & 24.4 & $23.5-25.2$ & 2 & 1.4 & \\
\hline \multicolumn{7}{|l|}{ Rib fractures } \\
\hline \multirow[t]{2}{*}{ Multiple (2) } & No & 25.0 & $23.4-28.1$ & 115 & 78.8 & \multirow[t]{2}{*}{0.78} \\
\hline & Yes & 25.5 & $22.5-27.8$ & 31 & 21.2 & \\
\hline \multirow[t]{2}{*}{ Multiple (3) } & No & 25.5 & $23.5-28.1$ & 121 & 82.9 & \multirow[t]{2}{*}{0.10} \\
\hline & Yes & 24.0 & $20.8-27.3$ & 25 & 17.1 & \\
\hline \multirow[t]{2}{*}{ Multiple $(\geq 4)$} & No & 25.0 & $22.5-27.7$ & 89 & 61.0 & \multirow[t]{2}{*}{0.24} \\
\hline & Yes & 25.2 & $24.0-28.4$ & 57 & 39.0 & \\
\hline \multicolumn{7}{|l|}{ Abdominal injury } \\
\hline \multirow[t]{2}{*}{ Intraabdominal } & No & 25.3 & $23.1-28.1$ & 121 & 82.9 & \multirow[t]{2}{*}{0.72} \\
\hline & Yes & 24.8 & $23.2-27.8$ & 25 & 17.1 & \\
\hline \multirow[t]{2}{*}{ Liver } & No & 25.1 & $23.1-28.1$ & 138 & 94.5 & \multirow[t]{2}{*}{0.71} \\
\hline & Yes & 25.8 & $22.4-27.6$ & 8 & 5.5 & \\
\hline \multirow[t]{2}{*}{ Spleen } & No & 25.2 & $22.7-27.8$ & 135 & 92.5 & \multirow[t]{2}{*}{0.55} \\
\hline & Yes & 24.9 & $23.8-33.2$ & 11 & 7.5 & \\
\hline \multirow[t]{2}{*}{ Duodenal } & No & 25.2 & $23.2-27.8$ & 145 & 99.3 & \multirow[t]{2}{*}{0.17} \\
\hline & Yes & 20.8 & $20.8-20.8$ & 1 & 0.7 & \\
\hline \multirow[t]{2}{*}{ Colon } & No & 25.2 & $23.1-27.8$ & 145 & 99.3 & \multirow[t]{2}{*}{0.82} \\
\hline & Yes & 24.7 & $24.7-24.7$ & 1 & 0.7 & \\
\hline Kidney & No & 25.2 & $23.1-27.8$ & 143 & 97.9 & 0.98 \\
\hline & Yes & 24.8 & $20.6-36.8$ & 3 & 2.1 & \\
\hline Bladder & No & 25.3 & $23.2-28.0$ & 144 & 98.6 & 0.24 \\
\hline & Yes & 22.7 & $20.6-24.7$ & 2 & 1.4 & \\
\hline
\end{tabular}

*Wilcoxon rank sum test 
mortality $\left(0(0 \%)\right.$ deaths for BMI $\geq 30 \mathrm{~kg} / \mathrm{m}^{2}$ vs $2(1.6 \%)$ deaths for BMI $\left.<30 \mathrm{~kg} / \mathrm{m}^{2}, p=1.00\right)$, rib fracture score (9 (42.9\%) RFS $>4$ for BMI vs $50(40.0 \%)$ RFS $\leq 4$ for $\mathrm{BMI}<30 \mathrm{~kg} / \mathrm{m}^{2}, p=0.56$ ) and abdominal injuries (liver: $p=0.23$, spleen: $p=0.21$, duodenum: $p=1.00$, colon: $p=1.00$, kidney: $p=0.38$, bladder: $p=0.46$ ). Similar results were observed when choosing a cut-off of $\geq 25 \mathrm{~kg}$ / $\mathrm{m}^{2}$.

The SOAF was not higher in patients who died (Table 2). It was increased in females, patients $>55$ years, and patients with comorbidity. When adjusting for the a priori confounder sex, this lack of association remained $\left(\mathrm{OR}_{\text {adjusted }}=2.8,95 \%\right.$ CI 1.03-7.72, $p=0.04)$. Patients with a liver injury did not have different values of SOAF. This association did not change in the logistic regression model $\left(\mathrm{OR}_{\text {adjusted }}=0.20\right.$, 95\% CI 0.04-0.98, $p=0.05$ ). An injury of the bladder was associated with lower values of SOAF (3.3 [IQR 2.8-4.1] vs 9.5 [IQR 6.5-14.4], $p=0.008$ ). This could not be fitted into a logistic regression model since all cases with a bladder rupture had $\mathrm{SPF}$ values $\leq 12.5$.

For SPF, no associations were seen with mortality (Table 3). However, lower values were found in males, patients aged $>55$ years, and patients with injuries of the bladder. The lack of association between a SPF $>12.5 \mathrm{~mm}$ and a liver injury remained in a logistic regression model $\left(\mathrm{OR}_{\text {adjusted }}=0.20,95 \%\right.$ CI $\left.0.04-0.94, p=0.042\right)$. Again, a logistic regression model for a bladder injury could not be fitted.

No association was found for UOAF and mortality (Table 4). Lower values of UOAF were associated with a liver injury. This association was weakened when applying the logistic regression model $\left(\mathrm{OR}_{\text {adjusted }}=0.21,95 \% \mathrm{CI}\right.$ $0.05-1.01, p=0.05)$.
Table 2 Association of outcome variables and subxiphoid outer abdominal fat (SOAF)

\begin{tabular}{|c|c|c|c|c|c|c|}
\hline \multirow[t]{2}{*}{ Variable } & \multirow[t]{2}{*}{ Category } & \multicolumn{4}{|c|}{ Subxiphoid outer abdominal fat (SOAF) $(n=248)$} & \multirow[t]{2}{*}{$p$ value* } \\
\hline & & Median & IQR & $n$ & $\%$ & \\
\hline \multirow[t]{2}{*}{ Sex } & Female & 13.1 & $8.0-18.8$ & 80 & 32.3 & \multirow[t]{2}{*}{$<0.001$} \\
\hline & Male & 8.4 & $5.6-12.3$ & 168 & 67.7 & \\
\hline \multirow[t]{2}{*}{ Age (years) } & $\leq 55$ & 7.9 & $5.5-13.8$ & 128 & 51.6 & \multirow[t]{2}{*}{0.009} \\
\hline & $>55$ & 10.8 & $7.4-14.9$ & 120 & 48.4 & \\
\hline \multirow[t]{2}{*}{ Comorbidity } & No & 7.8 & $5.2-11.9$ & 107 & 43.1 & \multirow[t]{2}{*}{$<0.001$} \\
\hline & Yes & 10.9 & $7.4-16.6$ & 141 & 56.9 & \\
\hline \multirow[t]{2}{*}{ Death } & No & 9.1 & $6.2-13.8$ & 226 & 91.1 & \multirow[t]{2}{*}{0.04} \\
\hline & Yes & 13.1 & $7.8-17.9$ & 22 & 8.9 & \\
\hline \multicolumn{7}{|l|}{ Rib fractures } \\
\hline \multirow[t]{2}{*}{ Multiple (2) } & No & 10.0 & $6.6-14.7$ & 197 & 79.4 & \multirow[t]{2}{*}{0.07} \\
\hline & Yes & 7.8 & $4.9-13.7$ & 51 & 20.6 & \\
\hline \multirow[t]{2}{*}{ Multiple (3) } & No & 10.1 & $6.5-13.9$ & 205 & 82.7 & \multirow[t]{2}{*}{0.78} \\
\hline & Yes & 8.8 & $6.2-15.7$ & 43 & 17.3 & \\
\hline \multirow[t]{2}{*}{ Multiple $(\geq 4)$} & No & 8.8 & $5.0-13.9$ & 139 & 56.0 & \multirow[t]{2}{*}{0.06} \\
\hline & Yes & 10.5 & $6.9-15.3$ & 109 & 44.0 & \\
\hline \multicolumn{7}{|l|}{ Abdominal injury } \\
\hline \multirow[t]{2}{*}{ Intraabdominal } & No & 10.1 & $6.5-14.6$ & 210 & 84.7 & \multirow[t]{2}{*}{0.15} \\
\hline & Yes & 8.1 & $5.5-12.1$ & 38 & 15.3 & \\
\hline \multirow[t]{2}{*}{ Liver } & No & 9.8 & $6.5-14.5$ & 237 & 95.6 & \multirow[t]{2}{*}{0.02} \\
\hline & Yes & 6.5 & $3.9-8.8$ & 11 & 4.4 & \\
\hline \multirow[t]{2}{*}{ Spleen } & No & 9.7 & $6.2-14.5$ & 231 & 93.1 & \multirow[t]{2}{*}{0.53} \\
\hline & Yes & 8.8 & $6.5-11.8$ & 17 & 6.9 & \\
\hline \multirow[t]{2}{*}{ Duodenal } & No & 9.5 & $6.2-14.4$ & 247 & 99.6 & \multirow[t]{2}{*}{0.98} \\
\hline & Yes & 9.3 & $9.3-9.3$ & 1 & 0.4 & \\
\hline \multirow[t]{2}{*}{ Colon } & No & 9.4 & $6.4-14.4$ & 247 & 99.6 & \multirow[t]{2}{*}{0.17} \\
\hline & Yes & 4.1 & $4.1-4.1$ & 1 & 0.4 & \\
\hline Kidney & No & 9.4 & $6.5-14.3$ & 244 & 98.4 & 0.58 \\
\hline & Yes & 9.3 & $4.4-13.6$ & 4 & 1.6 & \\
\hline Bladder & No & 9.5 & $6.5-14.4$ & 245 & 98.8 & 0.008 \\
\hline & Yes & 3.3 & $2.8-4.1$ & 3 & 1.2 & \\
\hline
\end{tabular}

*Wilcoxon rank sum test 
Table 3 Association of outcome variables and subxiphoid peritoneal fat (SPF)

\begin{tabular}{|c|c|c|c|c|c|c|}
\hline \multirow[t]{2}{*}{ Variable } & \multirow[t]{2}{*}{ Category } & \multicolumn{4}{|c|}{ Subxiphoid peritoneal fat (SPF) $(n=248)$} & \multirow[t]{2}{*}{$p$ value* } \\
\hline & & Median & IQR & $n$ & $\%$ & \\
\hline \multirow[t]{2}{*}{ Sex } & Female & 10.6 & $8.2-14.5$ & 80 & 32.3 & \multirow[t]{2}{*}{0.004} \\
\hline & Male & 13.2 & $9.5-17.2$ & 168 & 67.7 & \\
\hline \multirow[t]{2}{*}{ Age (years) } & $\leq 55$ & 10.9 & $7.9-15.1$ & 128 & 51.6 & \multirow[t]{2}{*}{0.003} \\
\hline & $>55$ & 13.4 & $10.1-17.2$ & 120 & 48.4 & \\
\hline \multirow[t]{2}{*}{ Comorbidity } & No & 12.7 & $8.2-16.1$ & 107 & 43.1 & \multirow[t]{2}{*}{0.77} \\
\hline & Yes & 12.3 & $9.3-16.5$ & 141 & 56.9 & \\
\hline \multirow[t]{2}{*}{ Death } & No & 12.4 & $8.8-16.3$ & 226 & 91.1 & \multirow[t]{2}{*}{0.99} \\
\hline & Yes & 13.1 & $9.9-14.7$ & 22 & 8.9 & \\
\hline \multicolumn{7}{|l|}{ Rib fractures } \\
\hline \multirow[t]{2}{*}{ Multiple (2) } & No & 12.8 & $9.3-16.2$ & 197 & 79.4 & \multirow[t]{2}{*}{0.13} \\
\hline & Yes & 10.6 & $7.8-15.9$ & 51 & 20.6 & \\
\hline \multirow[t]{2}{*}{ Multiple (3) } & No & 12.5 & $9.2-16.4$ & 205 & 82.7 & \multirow[t]{2}{*}{0.38} \\
\hline & Yes & 12.1 & $7.9-15.6$ & 43 & 17.3 & \\
\hline \multirow[t]{2}{*}{ Multiple $(\geq 4)$} & No & 12.3 & $8.1-15.7$ & 139 & 56.0 & \multirow[t]{2}{*}{0.12} \\
\hline & Yes & 12.8 & $9.5-16.6$ & 109 & 44.0 & \\
\hline \multicolumn{7}{|l|}{ Abdominal injury } \\
\hline \multirow[t]{2}{*}{ Intraabdominal } & No & 12.8 & $9.2-16.4$ & 210 & 84.7 & \multirow[t]{2}{*}{0.11} \\
\hline & Yes & 10.5 & $7.9-14.4$ & 38 & 15.3 & \\
\hline \multirow[t]{2}{*}{ Liver } & No & 12.7 & $9.1-16.3$ & 237 & 95.6 & \multirow[t]{2}{*}{0.03} \\
\hline & Yes & 9.8 & $5.9-12.5$ & 11 & 4.4 & \\
\hline \multirow[t]{2}{*}{ Spleen } & No & 12.7 & $9.0-16.3$ & 231 & 93.1 & \multirow[t]{2}{*}{0.50} \\
\hline & Yes & 11.0 & $8.3-14.3$ & 17 & 6.9 & \\
\hline \multirow[t]{2}{*}{ Duodenal } & No & 12.5 & $8.8-16.2$ & 247 & 99.6 & \multirow[t]{2}{*}{0.79} \\
\hline & Yes & 11.0 & $11.0-11.0$ & 1 & 0.4 & \\
\hline \multirow[t]{2}{*}{ Colon } & No & 12.5 & $9.0-16.2$ & 247 & 99.6 & \multirow[t]{2}{*}{0.12} \\
\hline & Yes & 5.3 & $5.3-5.3$ & 1 & 0.4 & \\
\hline Kidney & No & 12.5 & $8.8-16.2$ & 244 & 98.4 & 0.93 \\
\hline & Yes & 12.6 & $9.2-17.5$ & 4 & 1.6 & \\
\hline Bladder & No & 12.6 & $9.1-16.2$ & 245 & 98.8 & 0.009 \\
\hline & Yes & 5.3 & $5.0-6.6$ & 3 & 1.2 & \\
\hline
\end{tabular}

*Wilcoxon rank sum test
For UVF, no associations were observed with mortality (Table 5). Higher values were seen in males and patients aged $>55$ years. Patients with multiple $(\geq 4)$ rib fractures and liver injuries showed lower values of UVF. In the logistic regression model, this association disappeared $\left(\mathrm{OR}_{\text {adjusted }}=1.67,95 \%\right.$ CI $0.99-2.81, p=0.06$, $\mathrm{OR}_{\text {adjusted }}=0.0895 \%$ CI $0.01-0.67, p=0.02$, respectively).

Rib fractures of two ribs were not associated with any of the measures of obesity ( $p=0.78$ for BMI, $p=0.07$ for SOAF, $p=0.13$ for SPF, $p=0.27$, and $p=0.87$ for UOAF).

\section{Levels of obesity}

The BMI showed moderate correlations with all CTbased measurements for the level of obesity $(r=0.51$
[ $p<0.001]$ for SOAF, $r=0.55[p<0.001]$ for SPF, $r=0.59$ [ $p<0.001]$ for UOAF, and $r=0.56[p<0.001]$ for UVF) (Table 6; Figs. 2, 3). Both variables for subcutaneous fat revealed a moderate correlation $(r=0.64$ $[p<0.001]$ for SOAF and UOAF) and both variables for peritoneal fat also manifested a moderate correlation $(r=0.53[p<0.001]$ for SPF and UVF). Variables for subcutaneous and peritoneal fat showed weak correlations (e.g., $r=0.37[p<0.001]$ for SOAF and SPF).

\section{Rib fracture score and mortality}

The unadjusted effect of a RFS $>4$ on mortality $\left(\mathrm{OR}_{\text {crude }}=5.9,95 \%\right.$ CI 1.9-18.3, $\left.p=0.001\right)$ remained 
Table 4 Association of outcome variables and umbilical outer abdominal fat (UOAF)

\begin{tabular}{|c|c|c|c|c|c|c|}
\hline \multirow[t]{2}{*}{ Variable } & \multirow[t]{2}{*}{ Category } & \multicolumn{4}{|c|}{ Umbilical outer abdominal fat (UOAF) $(n=242)$} & \multirow[t]{2}{*}{$p$ value* } \\
\hline & & Median & IQR & $n$ & $\%$ & \\
\hline \multirow[t]{2}{*}{ Sex } & Female & 19.9 & 14.4 & 79 & 32.6 & \multirow[t]{2}{*}{0.42} \\
\hline & Male & 18.7 & $13.3-24.6$ & 163 & 67.4 & \\
\hline \multirow[t]{2}{*}{ Age (years) } & $\leq 55$ & 20.7 & $13.5-26.7$ & 126 & 52.1 & \multirow[t]{2}{*}{0.20} \\
\hline & $>55$ & 18.7 & $13.7-23.3$ & 116 & 47.9 & \\
\hline \multirow[t]{2}{*}{ Comorbidity } & No & 19.5 & $12.5-24.6$ & 105 & 43.4 & \multirow[t]{2}{*}{0.50} \\
\hline & Yes & 19.5 & $14.4-25.2$ & 137 & 56.6 & \\
\hline \multirow[t]{2}{*}{ Death } & No & 19.5 & $13.3-25.0$ & 221 & 91.3 & \multirow[t]{2}{*}{0.64} \\
\hline & Yes & 20.2 & $16.3-23.4$ & 21 & 8.7 & \\
\hline \multicolumn{7}{|l|}{ Rib fractures } \\
\hline \multirow[t]{2}{*}{ Multiple (2) } & No & 20.2 & $14.1-25.1$ & 192 & 79.3 & \multirow[t]{2}{*}{0.27} \\
\hline & Yes & 18.1 & $11.7-24.7$ & 50 & 20.7 & \\
\hline \multirow[t]{2}{*}{ Multiple (3) } & No & 19.5 & $13.5-25.5$ & 199 & 82.2 & \multirow[t]{2}{*}{0.98} \\
\hline & Yes & 19.9 & $13.2-24.9$ & 43 & 17.8 & \\
\hline \multirow[t]{2}{*}{ Multiple $(\geq 4)$} & No & 18.8 & $13.1-24.7$ & 136 & 56.2 & \multirow[t]{2}{*}{0.21} \\
\hline & Yes & 21.0 & $13.7-26.3$ & 106 & 43.8 & \\
\hline \multicolumn{7}{|l|}{ Abdominal injury } \\
\hline \multirow[t]{2}{*}{ Intraabdominal } & No & 20.2 & $13.9-25.7$ & 205 & 84.7 & \multirow[t]{2}{*}{0.04} \\
\hline & Yes & 16.7 & $11.7-22.4$ & 37 & 15.3 & \\
\hline \multirow[t]{2}{*}{ Liver } & No & 19.9 & $13.6-25.5$ & 231 & 95.5 & \multirow[t]{2}{*}{0.010} \\
\hline & Yes & 13.9 & $10.4-18.1$ & 11 & 4.5 & \\
\hline \multirow[t]{2}{*}{ Spleen } & No & 19.7 & $13.6-25.3$ & 226 & 93.4 & \multirow[t]{2}{*}{0.34} \\
\hline & Yes & 16.8 & $12.0-23.4$ & 16 & 6.6 & \\
\hline \multirow[t]{2}{*}{ Duodenal } & No & 19.5 & $13.5-25.0$ & 241 & 99.6 & \multirow[t]{2}{*}{0.69} \\
\hline & Yes & 16.9 & $16.9-16.9$ & 1 & 0.4 & \\
\hline \multirow[t]{2}{*}{ Colon } & No & 19.5 & $13.5-25.0$ & 241 & 99.6 & \multirow[t]{2}{*}{0.42} \\
\hline & Yes & 13.9 & $13.9-13.9$ & 1 & 0.4 & \\
\hline Kidney & No & 19.6 & $13.6-25.0$ & 238 & 98.3 & 0.23 \\
\hline & Yes & 11.6 & $5.3-25.3$ & 4 & 1.7 & \\
\hline Bladder & No & 19.6 & $13.5-25.2$ & 239 & 98.8 & 0.10 \\
\hline & Yes & 13.9 & $3.8-17.6$ & 3 & 1.2 & \\
\hline
\end{tabular}

*Wilcoxon rank sum test after fitting a logistic regression model for the a priori confounders sex and comorbidities $\left(\mathrm{OR}_{\text {adjusted }}=5.9,95 \%\right.$ CI 1.9-16.6, $p=0.002$ ) (Table 7; Fig. 4). The area under the curve was 0.75 (95\% CI 0.69-0.80). The chosen cutoff point for mortality was a RFS of 6 (sensitivity of $72.7 \%$ and specificity of $67.9 \%$ ).

\section{Discussion}

One of the main findings of this study about patients with rib fractures is the association between a RFS $>4$ and mortality. The global and local measures of obesity were not associated with mortality, rib fractures of liver injuries.
The BMI correlated moderately well with the CT-based measures of obesity.

The logistic regression model successfully controlled for sex and comorbidities. It would have been desirable to control for ISS, which had a median value of 25 (IQR 17.0-41.0). However, ISS was only available for 101 patients. This would have led to a substantial reduction of the sample size (from $n=259$ to $n=101$ ) and loss of power. Nonetheless, addition of the ISS to the logistic regression model would have reduced the effect of the RFS $>4$ on mortality in the reduced sample $\left(\mathrm{OR}_{\text {adjusted }}=4.0,95 \% \mathrm{CI}\right.$ $0.9-16.9, p=0.07)$. Therefore, future studies may include the ISS as a potential confounder in their logistic regression analysis. 
Table 5 Association of patient characteristics and outcome variables and umbilical visceral fat (UVF)

\begin{tabular}{|c|c|c|c|c|c|c|}
\hline \multirow[t]{2}{*}{ Variable } & \multirow[t]{2}{*}{ Category } & \multicolumn{4}{|c|}{ Umbilical visceral fat (UVF) $(n=242)$} & \multirow[t]{2}{*}{$p$ value* } \\
\hline & & Median & IQR & $n$ & $\%$ & \\
\hline \multirow[t]{2}{*}{ Sex } & Females & 49.5 & $37.6-63.0$ & 79 & 32.6 & \multirow[t]{2}{*}{$<0.001$} \\
\hline & Males & 64.7 & $49.7-84.0$ & 163 & 67.4 & \\
\hline \multirow[t]{2}{*}{ Age (years) } & $\leq 55$ & 57.2 & $42.1-76.2$ & 126 & 52.1 & \multirow[t]{2}{*}{0.007} \\
\hline & $>55$ & 62.1 & $47.6-88.5$ & 116 & 47.9 & \\
\hline \multirow[t]{2}{*}{ Comorbidities } & No & 58.7 & $44.8-80.5$ & 105 & 43.4 & \multirow[t]{2}{*}{0.42} \\
\hline & Yes & 59.3 & $44.0-82.6$ & 137 & 56.6 & \\
\hline \multirow[t]{2}{*}{ Death } & No & 59.3 & $44.0-81.1$ & 221 & 91.3 & \multirow[t]{2}{*}{0.89} \\
\hline & Yes & 57.9 & $44.9-78.6$ & 21 & 8.7 & \\
\hline \multicolumn{7}{|l|}{ Rib fractures } \\
\hline \multirow[t]{2}{*}{ Multiple (2) } & No & 58.7 & $44.8-81.2$ & 192 & 79.3 & \multirow[t]{2}{*}{0.87} \\
\hline & Yes & 62.2 & $42.9-79.8$ & 50 & 20.7 & \\
\hline \multirow[t]{2}{*}{ Multiple (3) } & No & 59.7 & $45.1-81.1$ & 199 & 82.2 & \multirow[t]{2}{*}{0.08} \\
\hline & Yes & 52.9 & $39.6-76.2$ & 43 & 17.8 & \\
\hline \multirow[t]{2}{*}{ Multiple $(\geq 4)$} & No & 55.8 & $41.8-77.7$ & 136 & 56.2 & \multirow[t]{2}{*}{0.010} \\
\hline & Yes & 53.6 & $49.0-82.0$ & 106 & 43.8 & \\
\hline \multicolumn{7}{|l|}{ Abdominal injury } \\
\hline \multirow[t]{2}{*}{ Intraabdominal } & No & 59.6 & $44.9-81.2$ & 205 & 84.7 & \multirow[t]{2}{*}{0.50} \\
\hline & Yes & 56.6 & $40.4-76.2$ & 37 & 15.3 & \\
\hline \multirow[t]{2}{*}{ Liver } & No & 59.7 & $44.9-81.2$ & 231 & 95.5 & \multirow[t]{2}{*}{0.006} \\
\hline & Yes & 44.0 & $37.1-56.6$ & 11 & 4.5 & \\
\hline \multirow[t]{2}{*}{ Spleen } & No & 59.0 & $43.9-81.1$ & 226 & 93.4 & \multirow[t]{2}{*}{0.57} \\
\hline & Yes & 61.4 & $54.6-78.5$ & 16 & 6.6 & \\
\hline \multirow[t]{2}{*}{ Duodenal } & No & 59.3 & $44.7-81.1$ & 241 & 99.6 & \multirow[t]{2}{*}{0.18} \\
\hline & Yes & 34.3 & $34.3-34.3$ & 1 & 0.4 & \\
\hline \multirow[t]{2}{*}{ Colon } & No & 59.3 & $44.7-81.1$ & 241 & 99.6 & \multirow[t]{2}{*}{0.13} \\
\hline & Yes & 29.6 & $29.6-29.6$ & 1 & 0.4 & \\
\hline Kidney & No & 59.0 & $44.0-81.1$ & 238 & 98.3 & 0.97 \\
\hline & Yes & 65.1 & $47.2-77.4$ & 4 & 1.7 & \\
\hline Bladder & No & 59.3 & $44.8-81.1$ & 239 & 98.8 & 0.03 \\
\hline & Yes & 40.4 & $29.6-40.8$ & 3 & 1.2 & \\
\hline
\end{tabular}

*Wilcoxon rank sum test

Table 6 Correlation of different measurement techniques for the level of obesity $(n=259)$

\begin{tabular}{llllll}
\hline Variable & \multicolumn{2}{l}{ Correlation coefficient $(p \text { value })^{*}$} & & \\
\cline { 2 - 6 } & BMI & SOAF & SPF & UOAF & UVF \\
\hline Body mass index (BMI) & 1.000 & $0.514(<0.001)$ & $0.546(<0.001)$ & $0.587(<0.001)$ & $0.561(<0.001)$ \\
Subxiphoid outer abdominal fat (SOAF) & $0.514(<0.001)$ & 1.000 & $0.369(<0.001)$ & $0.637(<0.001)$ & $0.319(<0.001)$ \\
Subxiphoid peritoneal fat (SPF) & $0.546(<0.001)$ & $0.369(<0.001)$ & 1.000 & $0.389(<0.001)$ & $0.532(<0.001)$ \\
Umbilical outer abdominal fat (UOAF) & $0.587(<0.001)$ & $0.637(<0.001)$ & $0.389(<0.001)$ & 1.000 & $0.290(<0.001)$ \\
Umbilical visceral fat (UVF) & $0.561(<0.001)$ & $0.319(<0.001)$ & $0.532(<0.001)$ & $0.290(<0.001)$ & 1.000 \\
\hline
\end{tabular}

*Spearman rank correlation coefficient

Previous studies found that rib fracture patients had higher risks of adverse outcomes, such as pneumonia and that increasing numbers of rib fractures were associated with mortality [7-9]. In 64,750 rib fracture patients from the National Trauma Data Bank (NTDB), the overall death rate was $10 \%$ [9]. When stratified according to the number of rib fractures, patients with only one rib fracture had a mortality of $5.8 \%$, while those with five rib fractures had a mortality of $9.9 \%$, and those with $\geq 8$ rib fractures had a mortality of $34.4 \%$. This is in line with the presented results since 


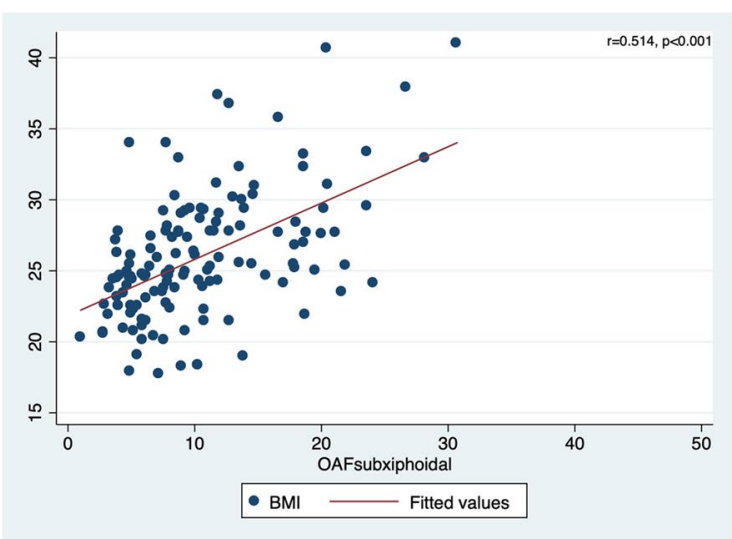

A

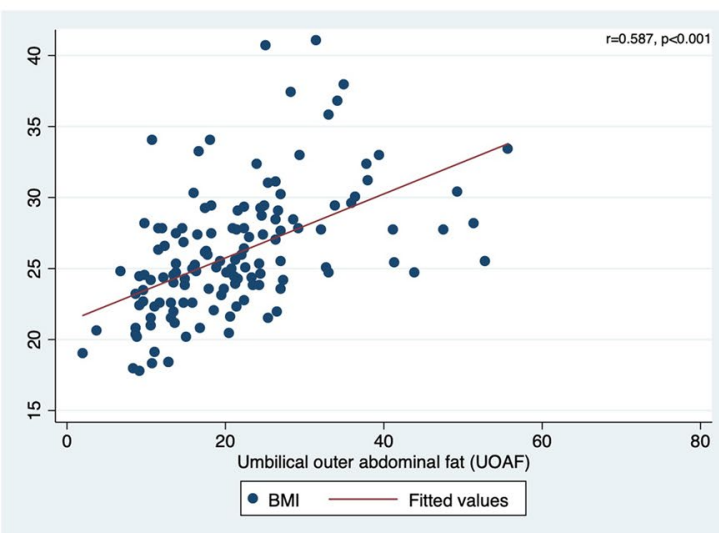

C

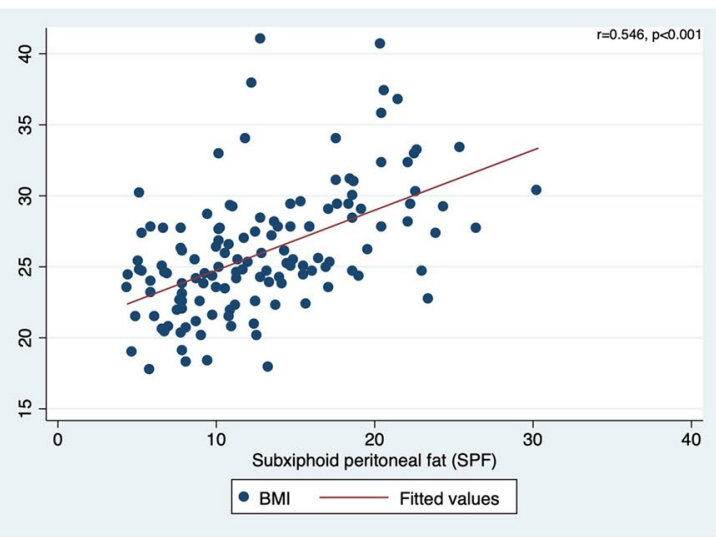

B

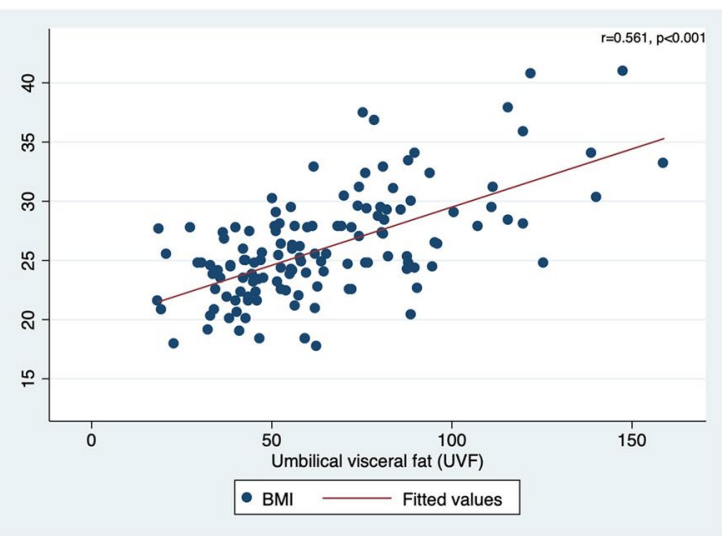

D

Fig. 2 Correlations of the body mass index (BMI) with all computed tomography (CT)-based measurements for the level of obesity. a Subxiphoid outer abdominal fat (SOAF). b Subxiphoid peritoneal fat (SPF). c Umbilical outer abdominal fat (UOAF). d Umbilical visceral fat (UVF)

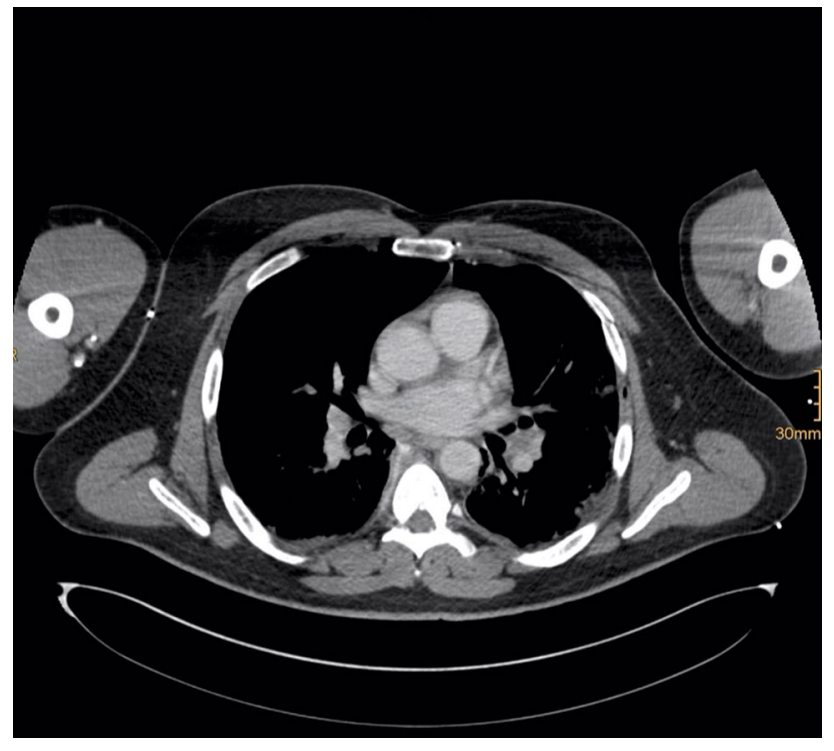

Fig. 3 Illustration of a 42-year-old male with a left-sided anterolateral $\mathrm{rib}$ fracture and $\mathrm{BMI} \geq 30 \mathrm{~kg} / \mathrm{m}^{2}$ an increased RFS $>4$ was associated with higher mortality. Contrarily, 2 recent retrospective studies, which included 594 and 1272 patients with rib fractures, did not observe an influence on morbidity in the intensive care unit (ICU) and mortality $[12,13]$. This may be explained by the adjustment for confounders in the present study. Furthermore, other scores than the RFS have been proposed, but the RFS score seems to be a useful and easy tool to account for the severity of rib fractures taking into account the patient's age [19].

A population-based cohort study of men $\geq 65$ years showed that multiple rib fractures were more common in obese patients (relative risk $=4.0,95 \%$ CI 1.2-13.5) [6]. In contrast, this association was not observed in postmenopausal women [11]. In the present study, no measures of obesity were associated with rib fractures. Therefore, the level of obesity and local fat does neither seem protective, nor a risk factor for rib fractures.

The BMI and waist circumferences are valuable tools for obesity, but they do lack a precise discernment of fat from other tissue as well as limited accuracy and reproducibility, 
Table 7 Unadjusted data and logistic regression model for the association of the rib score with mortality $(n=259)$

\begin{tabular}{|c|c|c|c|c|c|c|c|}
\hline \multirow[t]{2}{*}{ Variable } & \multirow[t]{2}{*}{ Category } & \multicolumn{3}{|l|}{ Unadjusted } & \multicolumn{3}{|l|}{ Adjusted* } \\
\hline & & OR & $95 \%$ CI & $p$ value $^{\dagger}$ & OR & $95 \%$ CI & $p$ value $e^{\ddagger}$ \\
\hline \multirow[t]{2}{*}{ Rib fracture score $(\mathrm{RFS})^{\S}$} & $\leq 4$ & 1.0 (reference) & & & 1.0 (reference) & & \\
\hline & $>4$ & 5.9 & $1.9-18.3$ & 0.001 & 5.9 & $1.9-16.6$ & 0.002 \\
\hline \multirow[t]{2}{*}{ Sex } & Female & 1.0 (reference) & & & 1.0 (reference) & & \\
\hline & Male & 0.7 & $0.3-1.7$ & 0.5 & 0.9 & $0.3-2.2$ & 0.74 \\
\hline \multirow[t]{2}{*}{ Comorbidities } & No & 1.0 (reference) & & & 1.0 (reference) & & \\
\hline & Yes & 1.4 & $0.6-3.5$ & 0.47 & 0.9 & $0.3-2.3$ & 0.80 \\
\hline
\end{tabular}

$O R$ odds ratio, $C I$ confidence interval

*Adjusted for all variables given in the table. Sex and comorbidities were considered as a priori confounders

${ }^{\dagger}$ Chi-squared test

${ }^{\ddagger}$ Wald test

${ }^{\S}$ Rib fracture score (RFS): number of rib fractures $\times$ side + age category (where side: unilateral $=1$ and bilateral $=2$; age category: $<50=0$, $50-60=1,61-70=2,71-80=3$, and $>80=4$ ) [17]

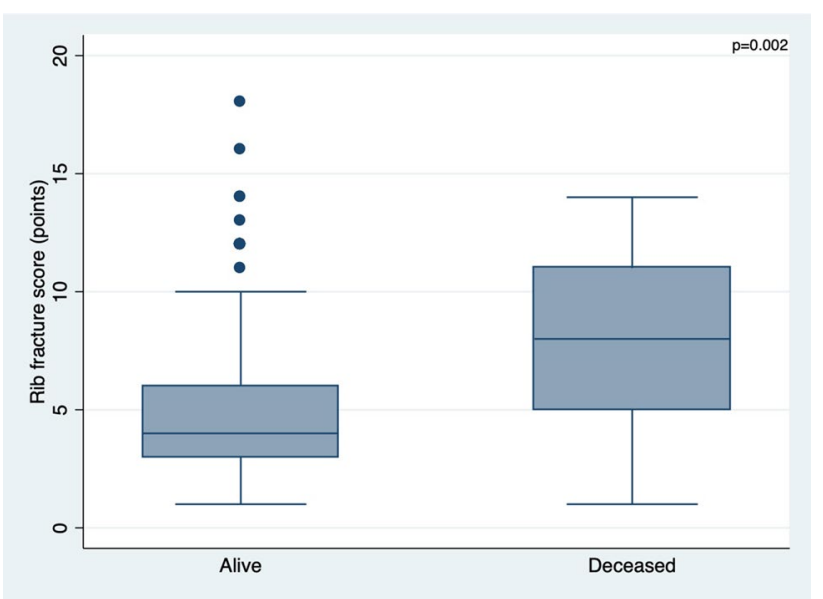

Fig. 4 Box plot showing the effect of a RFS $>4$ on mortality ( $p$ value is adjusted for sex and comorbidities)

respectively [17]. A large collaborate report of 57 prospective studies stated that it can be used as a predictor of all-cause mortality [2]. The survival was reduced by up to 4 years if $>30-35 \mathrm{~kg} / \mathrm{m}^{2}$ and up to 10 years if $>40-45 \mathrm{~kg}$ / $\mathrm{m}^{2}$. A recent systematic review and meta-analysis by Kinder et al. reported the effect of obesity on Orthopaedic Trauma patients [4]. In 379,333 patients, complications were more common in obese patients [OR for complications of 2.32 ( $23 \%$ complications if $\mathrm{BMI} \geq 30$ versus $14 \%$ if $\mathrm{BMI}<25$ )]. Obesity was also associated with higher mortality, more infections, non-union tibial fractures, and thrombosis. On the other hand, another meta-analysis suggested the "obesity paradox" indicating that obesity may provide some protection against certain injuries due to a cushioning effect and higher regional bone mineral density, such as hip fractures
[5]. In 3,126,313 patients, obese patients showed significantly decreased hip fracture risk. Other studies have concluded that obesity does not have a protective effect against fractures [23, 24]. Our study adds to these findings in that there was no observed difference in mortality, rib fracture severity, and abdominal injuries between obese and nonobese patients. Theoretically, BMI may not be an ideal parameter for the "obesity paradox". In another prospective investigation of a large cohort, the waist circumference was also associated with increased overall mortality [3]. In their study, the correlation between the BMI and waist circumference was very strong $(r=0.85)$. The reason for only moderate correlations with the BMI is difficult to explain. Potentially, the supine position and location of measurements at the subxiphoid and umbilical level may have played a role. One option to overcome this limitation in the future may be to use volumetric measurements instead of distances [18]. In a trauma setting, obtaining the BMI at the initial stage does not seem very feasible because patients are usually in a supine position and measuring the weight and height would be complicated. The waist circumference could be measured more easily, but according to our knowledge, this is not routinely done. This could be attributed to the fact that the focus is set on essential investigations in the resuscitation room according to the algorithm of the advanced trauma life support (ATLS). Evaluating CT-based measures for obesity is a handy option for easy assessment at any time. These could also be studied using ultrasonography [14]. In a study of 26 non-obese patients, strong correlations as well as excellent intra- and interobserver agreement were found between CTand ultrasonography-based measurements for subcutaneous and visceral measurements [17]. 


\section{Conclusions}

In this patient cohort, a RFS $>4$ was an independent risk factors for increased mortality. Global and local measures of obesity were not associated with mortality, rib fractures or liver injuries. If the BMI is not available in trauma patients, CT-based measures of obesity may be considered as a surrogate for further evaluation.

Acknowledgements The abstract was presented as a poster presentation at the annual congress of orthopaedics and traumatology (DKOU), Berlin, Germany, October 24-27, 2017.

Author contributions TJ: idea, conception and design, ethical approval, statistical analysis and interpretation of data, drafting the manuscript; RMM: acquisition of data; BS: advice on statistical analysis; $\mathrm{VN}$ and HPS: interpretation of data; CEWS: acquisition and interpretation of data. CMLW: ethical approval, interpretation of data. All: revision of the manuscript, final approval of the version to be published.

Funding Open access funding provided by University of Zurich.

\section{Compliance with ethical standards}

Conflict of interest The authors declare that they have no conflict of interests.

Open Access This article is licensed under a Creative Commons Attribution 4.0 International License, which permits use, sharing, adaptation, distribution and reproduction in any medium or format, as long as you give appropriate credit to the original author(s) and the source, provide a link to the Creative Commons licence, and indicate if changes were made. The images or other third party material in this article are included in the article's Creative Commons licence, unless indicated otherwise in a credit line to the material. If material is not included in the article's Creative Commons licence and your intended use is not permitted by statutory regulation or exceeds the permitted use, you will need to obtain permission directly from the copyright holder. To view a copy of this licence, visit http://creativecommons.org/licenses/by/4.0/.

\section{References}

1. Sharma OP, Oswanski MF, Jolly S, Lauer SK, Dressel R, Stombaugh HA. Perils of rib fractures. Am Surg. 2008;74(4):310-4.

2. Whitlock G, Lewington S, Sherliker P, Clarke R, Emberson J, Halsey J, et al. Body-mass index and cause-specific mortality in 900000 adults: collaborative analyses of 57 prospective studies. Lancet. 2009;373(9669):1083-96.

3. Pischon T, Boeing H, Hoffmann K, Bergmann M, Schulze MB, Overvad K, et al. General and abdominal adiposity and risk of death in Europe. N Engl J Med. 2008;359(20):2105-20.

4. Kinder F, Giannoudis PV, Boddice T, Howard A. The effect of an abnormal BMI on orthopaedic trauma patients: a systematic review and meta-analysis. J Clin Med. 2020;9(5):1302.

5. Tang X, Liu G, Kang J, Hou Y, Jiang F, Yuan W, et al. Obesity and risk of hip fracture in adults: a meta-analysis of prospective cohort studies. PLoS ONE. 2013;8(4):e55077.

6. Premaor MO, Compston JE, Fina Avilés F, Pagès-Castellà A, Nogués X, Díez-Pérez A, et al. The association between fracture site and obesity in men: a population-based cohort study. J Bone Miner Res. 2013;28(8):1771-7.
7. Dunham CM, Hileman BM, Ransom KJ, Malik RJ. Trauma patient adverse outcomes are independently associated with rib cage fracture burden and severity of lung, head, and abdominal injuries. Int J Burns Trauma. 2015;5(1):46-55.

8. Byun JH, Kim HY. Factors affecting pneumonia occurring to patients with multiple rib fractures. Korean J Thorac Cardiovasc Surg. 2013;46(2):130-4.

9. Flagel BT, Luchette FA, Reed RL, Esposito TJ, Davis KA, Santaniello JM, et al. Half-a-dozen ribs: the breakpoint for mortality. Surgery. 2005;138(4):717-23 (discussion 23-25).

10. Center JR, Nguyen TV, Schneider D, Sambrook PN, Eisman JA. Mortality after all major types of osteoporotic fracture in men and women: an observational study. Lancet. 1999;353(9156):878-82.

11. Prieto-Alhambra D, Premaor MO, Fina Avilés F, Hermosilla E, Martinez-Laguna D, Carbonell-Abella C, et al. The association between fracture and obesity is site-dependent: a populationbased study in postmenopausal women. J Bone Miner Res. 2012;27(2):294-300.

12. Söderlund T, Ikonen A, Pyhältö T, Handolin L. Factors associated with in-hospital outcomes in 594 consecutive patients suffering from severe blunt chest trauma. Scand J Surg. 2015;104(2):115-20.

13. Lin FC, Li RY, Tung YW, Jeng KC, Tsai SC. Morbidity, mortality, associated injuries, and management of traumatic rib fractures. J Chin Med Assoc. 2016;79(6):329-34.

14. Mook-Kanamori DO, Holzhauer S, Hollestein LM, Durmus B, Manniesing R, Koek M, et al. Abdominal fat in children measured by ultrasound and computed tomography. Ultrasound Med Biol. 2009;35(12):1938-46.

15. Gur EB, Ince O, Turan GA, Karadeniz M, Tatar S, Celik E, et al. Ultrasonographic visceral fat thickness in the first trimester can predict metabolic syndrome and gestational diabetes mellitus. Endocrine. 2014;47(2):478-84.

16. Jentzsch T, Geiger J, Slankamenac K, Werner CM. Obesity measured by outer abdominal fat may cause facet joint arthritis at the lumbar spine. J Back Musculoskelet Rehabil. 2015;28(1):85-91.

17. Bazzocchi A, Filonzi G, Ponti F, Sassi C, Salizzoni E, Battista G, et al. Accuracy, reproducibility and repeatability of ultrasonography in the assessment of abdominal adiposity. Acad Radiol. 2011;18(9):1133-43.

18. Fox CS, Massaro JM, Hoffmann U, Pou KM, Maurovich-Horvat $\mathrm{P}$, Liu CY, et al. Abdominal visceral and subcutaneous adipose tissue compartments: association with metabolic risk factors in the Framingham Heart Study. Circulation. 2007;116(1):39-48.

19. Easter A. Management of patients with multiple rib fractures. Am J Crit Care. 2001;10(5):320-7 (quiz 8-9).

20. Maxwell CA, Mion LC, Dietrich MS. Hospitalized injured older adults: clinical utility of a rib fracture scoring system. J Trauma Nurs. 2012;19(3): 168-74 (quiz 75-76).

21. Jentzsch T, Neuhaus V, Seifert B, Osterhoff G, Simmen HP, Werner CM, et al. The impact of public versus private insurance on trauma patients. J Surg Res. 2016;200(1):236-41.

22. World Health Organization (WHO). International statistical classification of diseases and related health problems (ICD-10). Geneva: World Health Organization (WHO). https://www.who. int/classifications/icd/ICD10Volume2_en_2010.pdf. Accessed 2 Sept 2020.

23. Lespessailles E, Paccou J, Javier RM, Thomas T, Cortet B, Committee GS. Obesity, bariatric surgery, and fractures. J Clin Endocrinol Metab. 2019;104(10):4756-68.

24. Nakamura KM, Haglind EG, Clowes JA, Achenbach SJ, Atkinson EJ, Melton LJ, et al. Fracture risk following bariatric surgery: a population-based study. Osteoporos Int. 2014;25(1):151-8. 